\title{
LANDKREISE
}

\section{Zwischen Markt und Hilfe}

\author{
Andreas Strunk
}

Theorie und Praxis der Sozialraumorientierung konzentrieren sich auf Städte und Stadtteile. Doch auch auf dem Land ist eine Sozialraumorientierung erforderlich. Wichtiger Kooperationspartner dabei ist der Allgemeine Soziale Dienst.

Die bisherige Praxiserfahrung bei der Sozialraumentwicklung wird stark aus der Perspektive großstädtischer Räume bestimmt - also aus der Perspektive jener Städte, die sowohl örtlicher Träger der Jugend- und Sozialhilfe sind als auch Verantwortung tragen für die Stadtentwicklungsplanung.

In Landkreisen ist das oft anders. Dort ist oft der Landkreis örtlicher Träger der Jugend- und Sozialhilfe und die Verantwortung für die Stadt- und Gemeindeentwicklung liegt bei den Kommunen, die im jeweiligen Landkreis liegen. In Deutschland gibt es circa 300 Landkreise. Dort wohnen rund 56 Millionen Einwohnerinnen und Einwohner. Die Landkreise bilden etwa 96 Prozent der Gesamtfläche der Bundesrepublik Deutschland. Probleme, die mit dem Auseinanderfallen von örtlicher Zuständigkeit der Jugend- und Sozialhilfe und gemeindlicher Zuständigkeit für die lokale Planung zusammenhängen können, betreffen also viele Bürgerinnen und Bürger im Land.

Bei Themen der Sozialraumorientierung spielt die Erfahrung der Fachkräfte aus den Allgemeinen Sozialen Diensten (ASD) eine große Rolle. Was ich mit »Auseinanderfallen « meine, das will ich an zwei Städten skizzieren: Stuttgart ist ein Stadtkreis. Stuttgart ist gleichzeitig örtlicher Träger der Jugend- und Sozialhilfe. Der Allgemeine Soziale Dienst dort ist Teil der Stadtverwaltung. Die Stadtverwaltung ist immer als eine »Gesamthandelnde« zu verstehen. Die Koordination aller für die Sozialraumentwicklung relevanten Tatbestände kann auf Dezernatsebene gelingen. Es gibt nur ein politisches Gremium für alle relevanten Probleme, den Gemeinderat.

Anders sieht es in Schwäbisch Gmünd aus. Schwäbisch Gmünd ist eine große Stadt im Landkreis. Der örtliche Träger der Jugend- und Sozialhilfe ressortiert beim Landkreis; also beim Landratsamt in Aalen. Der Allgemeine Soziale Dienst ist hier nicht Teil der eigenen Stadtverwaltung. In Schwäbisch Gmünd gibt es lediglich eine Außenstelle. Die Kommune hat aber eine Zuständigkeit für alle Themen der Stadtverwaltung. Es gibt keine institutionalisierte Schnittstelle zur Arbeit des Allgemeinen Sozialen Dienstes und relevante Fragen sind in zwei parlamentarischen Gremien zu erörtern: Kreistag und Gemeinderat.

Was bedeutet das für diesen Fall des Auseinanderfallens? Viele Wissenstatbestände des Allgemeinen Sozialen

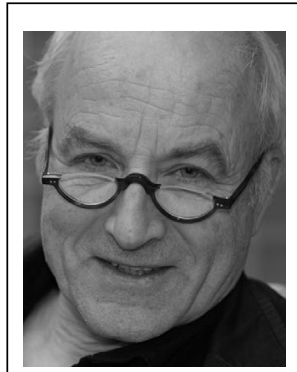

Prof. Dr. Andreas Strunk gehört zu den Mitbegründern von SOZIALwirtschaft zu Beginn der 1990er Jahre. Noch heute ist er Mitglied im Beirat der Zeitschrift SOZIALwirtschaft und des Informationsdienstes SOZIALwirtschaft aktuell. Er war bis zu seiner Pensionierung

Hochschullehrer an der Fachhochschule Esslingen und vertrat dort die Fächer Sozialpädagogik und Organisationswissen für die Soziale Arbeit. Zurzeit ist er als Berater für die Gesellschaft für Innovation, Systementwicklung und Soziale Arbeit (GISAmbH) tätig. E-Mail prof.strunk@t-online.de

Dienstes, der beim Landratsamt ressortiert, können auf kommunaler Ebene nicht genutzt werden. Das ist bedauerlich, weil beim Allgemeinen Sozialen Dienst in der Regel viele relevante Informationen vorliegen, die für die kommunale Entwicklung relevant sein können. Beispiele können sein: Kenntnisse über

- die Lebenslage alter Menschen in Beständen, die im Rahmen von Wohnungsprivatisierung verkauft worden sind

- die Versorgungssituation von Risikohaushalten auf dem Normalmarkt des Wohnens

- die Konzentration belasteter Familien in bestimmten Straßenzügen und Quartieren

- die Qualität von Spiel- und Erlebnisräumen für Kinder und Jugendliche

- die Isolation von Frauen und Mädchen mit Migrationshintergrund und die Konsequenzen für die jeweilige Familiendynamik

- die Überbelegung in Wohnungen

- die Mängel in der Versorgungslage alter Menschen (Überversorgung im stationären Bereich, Unterversorgung im offenen Bereich, Probleme in Siedlungen aus den 1950er-Jahren usf.)

- die Betreuungsprobleme bei sogenannten Lückekindern.

Diese Liste könnte sicher jeder Mitarbeitende aus dem jeweiligen Allgemeinen Sozialen Dienst ergänzen. Das Wissen des Allgemeinen Sozialen Dienstes könnte im Hinblick auf Themen der Stadtentwicklung und damit der Sozialraumentwicklung genutzt werden als Bestandteil

- eines Frühwarnsystems

- von Zielgruppen-Fachplänen 
- von Stadtentwicklungsplänen im engeren Sinne

- von Evaluationsvorhaben für kommunale Pläne.

Die Nutzung entsprechender Wissenstatbestände gelingt in den Kommunen besser, die gleichzeitig örtlicher Träger der Sozial- und Jugendhilfe sind. Die gesamte Sozialraumdebatte konzentriert sich seit Jahren auf großstädtische Erfahrungsfelder. Die Schwierigkeiten der Entwicklung einer sozialraumorientierten Politik in den Landkreisen wird nach meiner Einschätzung nicht ausreichend thematisiert.

Wie kann man es besser machen? Dazu will ich sechs Überlegungen anbieten: erstens die Problematisierung unterschiedlicher Sozialraumorientierungs-Konzepte, zweitens die Entschlossenheit auf kommunaler Ebene, drittens die Landkreisplanung als Dienstleistung für kreisangehörige Kommunen, viertens die Wissensbilanzierung und Netzwerkmanagement, fünftens die Arbeit mit Projektstrukturen und sechstens die Schaffung integrierter Datensätze.

\section{Erstens: Problematisierung unterschiedlicher Sozial-} raumorientierungs-Konzepte

Auf kommunaler Ebene muss der folgende Sachverhalt zunächst problematisiert werden. Sozialraumorientierung aus kommunaler Sicht im Bereich der Stadtentwicklung ist nicht zwingend deckungsgleich mit Sozialraumorientierung aus der Sicht der örtlichen Träger der Jugend- und Sozialhilfe. Bei der Stadt- und Gemeindeplanung geht es vor allen Dingen um Dominanten des Marktes. Relevant sind vor allem solche Sachverhalte wie: Bodenpreise, Investorenvorlieben, vorhandene Grundstücke, Nachfrageorientierung in der Wohnungspolitik etc. Bei der Nachfrageorientierung geht es immer um generalisierbare Sachverhalte, also prinzipiell nicht um eine Einzelfallorientierung. $\mathrm{Zu}$ beachten ist auch die Planerinnen- und Planerprofessionalisierung in dem Bereich der kommunalen Stadtentwicklungsplanung. Die Planerinnen und Planer orientieren sich in der Regel nur nachrangig an den Themen des Sozialen, was auch immer das heißen mag.

Sozialraumorientierung im Verständnis von Jugendund Sozialhilfe meint vor allem Lebensfeldorientierung: Welche Ressourcen sind vor Ort aktivierbar, um die Lebenslage von problembelasteten Kindern, Jugendlichen und Erwachsenen zu verbessern? Natürlich gibt es Zusammenhänge und gemeinsame Ziele, aber die Durchsetzbarkeit dessen, was Sozial- und Jugendhilfe will, ist schwierig und oft prinzipiell nicht möglich. Das hängt vor allem mit den Machtverhältnissen der beteiligten Ämter und Dezernate zusammen.

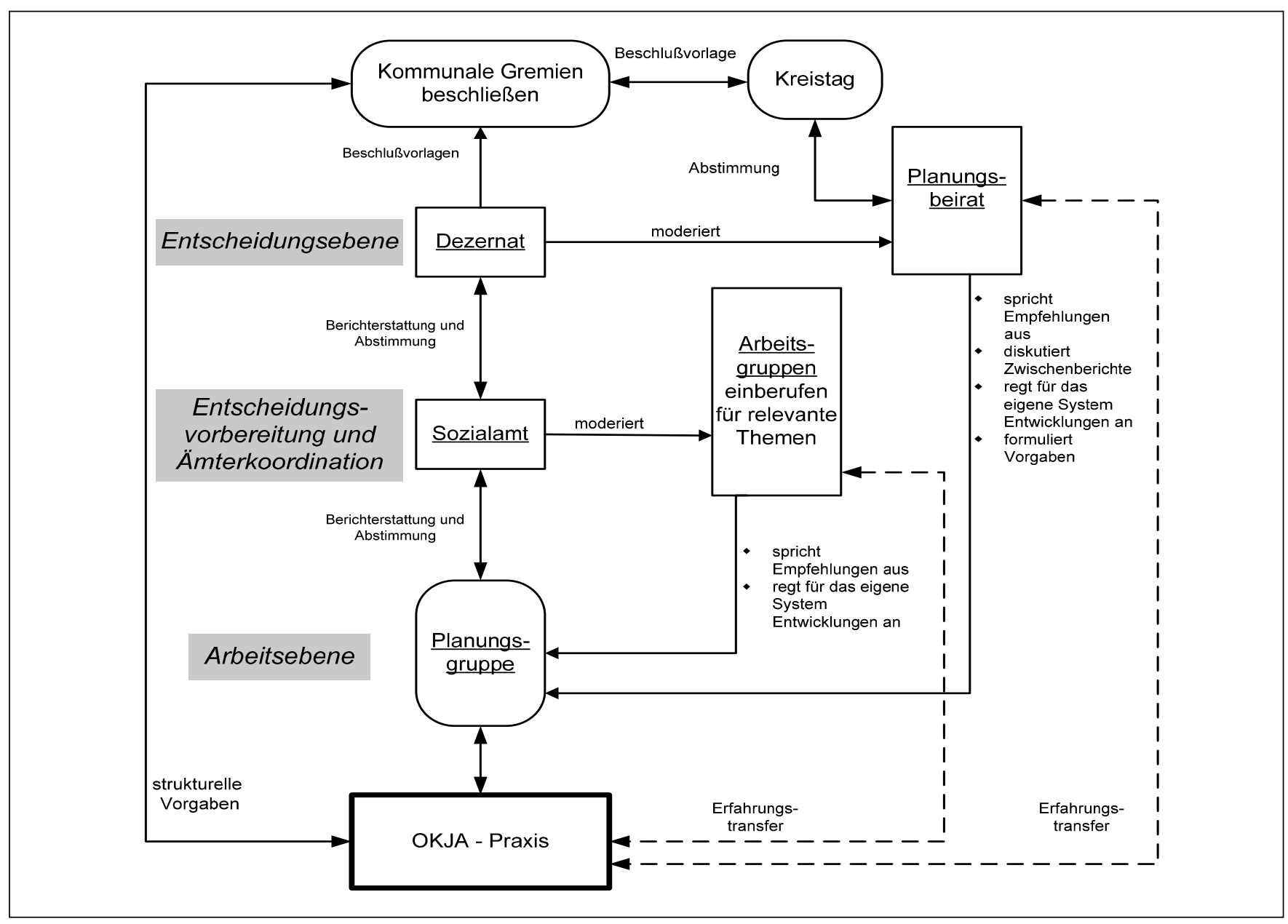

Abb. Projektstruktur: Eine Kommune muss dafür sorgen, dass genutzt werden kann, was der überordnete Landkreis weiß. Das Beispiel aus Schwäbisch Gmünd zeigt, wie eine solche Projektstruktur zur Fortentwicklung der »offenen Kinder- und Jugendarbeit» (OKJA) organisiert werden kann. 


\section{Wie man Sozialräume untersuchen kann}

Gelingendes Leben im Alltag ist $u$. a. angewiesen auf eine angemessene Ausstattung des Raumes, in dem die Menschen leben. Insofern brauchen Politik und Verwaltung verlässliche Indikatoren, um die Qualität der Lebensräume beurteilen zu können, für die sie Verantwortung tragen und die sie durch geeignete Maßnahmen entwickeln können. In diesem Zusammenhang haben sich Instrumente der Sozialraumanalyse bewährt.

Die Sozialraumanalyse liefert relevante Daten für die Sozialplanung. Der Erfolg der Sozialplanung muss abschließend (nach Umsetzung entsprechender Maßnahmen) überprüft werden. Dies ist die Aufgabe einer Wirkungskontrolle. Im Rahmen der Wirkungskontrolle können auch Aussagen gemacht werden über die Brauchbarkeit der Indikatoren, die in der Sozialraumanalyse benutzt wurden. Eine Reihe von Fragen müssen für eine qualifizierte Sozialraumanalyse und Sozialplanung beantwortet werden:

- Wie kommt man zu verlässlichen Indikatoren für die Beurteilung lebensweltlicher Qualität?

- Wie müssen die Ergebnisse der Sozialraumanalyse aufbereitet werden für örtliche Aushandlungs- und Bewertungsprozesse?

- Wie sind die Bewohnerinnen und Bewohner zu beteiligen unter Beachtung der Stärkung ihrer eigenen Handlungsmöglichkeiten?

- Welche Verfahren für ein Monitoring sind geeignet?

- Wie kann Sozialraumanalyse die fachliche, räumliche und finanzielle Planung bedienen?

Folgende Handlungsempfehlungen müssen beachtet werden:

- Notwendig ist ein politischer Grundsatzbeschluss zur Implementation des gesamten Verfahrens.

- Es sind die entsprechenden fachlichen Strukturen sowohl qualitativ als auch quantitativ zu schaffen.
- Die Fachkräfte der Sozialraumanalyse und der Sozialplanung stehen in einer tragfähigen Kooperation mit allen relevanten Akteuren im Sozialraum.

- Garantiert wird eine ergebnisoffene und prozesshafte Organisation der Planung.

- Förderinstrumente aus übergeordneten Politikbereichen (z. B. Europäische Union und Bund) werden integriert und genutzt.

- Vereinbart wird eine Wirkungskontrolle über Maßnahmen, die auf einer Sozialraumanalyse basieren.

Auf diese Weise bekommt die Sozialverwaltung als Träger von Sozialraumanalyse und Sozialplanung eine Mittelpunktsfunktion in der Stadt, sie kann sich als Treiber einer sozialen Kommunalpolitik profilieren und »eine starke Achse bilden, wenn es darum geht, die Sozialraumentwicklung an einem runden Tisch qualifiziert voranzubringen und die Potenziale, quersektoriell über Träger, Einrichtungen, Gruppen, Professionen und Köpfe zu bündeln. Interdisziplinäre Kooperation heißt hier, Kompetenzen von anderer Seite beiholen, von anderen lernen, gemeinsam lernen.«(1) Inzwischen gibt es umfangreiche Erfahrungen zum Instrument Sozialraumanalyse. (2) Vor allem für den Bereich der Jugendhilfeplanung gibt es gute Beispiele. (3)

\section{Andreas Strunk}

(1) Verein für Sozialplanung e. V., Fachpolitische Stellungnahme »Sozialraumanalyse und Sozialraumplanung «

(2) 1998; Riege, Marlo, Herbert Schubert (Hg.). Sozialraumanalyse, 2005.

(3) Jordan, E., Schone, R., Jugendhilfeplanung, 1992.

Quelle: Bernd Maelicke (Hg.): Lexikon der Sozialwirtschaft. Nomos Verlagsgesellschaft, Baden-Baden 2007. 1.128 Seiten. 98,- Euro. ISBN 978-3-8329-2511-6. Seite 943. 
Man muss unterscheiden zwischen Faktoren einer marktgetriebenen Sozialraumorientierung (Markt, Boden, Kapital, Investoren) und den Faktoren einer hilfreichen Sozialraumorientierung (Subsidiarität, Lebenswelt, Ressourcen, gelingender Alltag, Unterstützung, Wohlfahrtsmix). Man kann die kommunalen Ämter unschwer den entsprechenden Selbstorganisationsfeldern zuordnen. In die marktgetriebene Sozialraumorientierung gehören Ämter wie: Stadtplanungsamt, Liegenschaftsamt, Baurechtsamt, Ordnungsamt. In den Bereich der hilfreichen Sozialraumorientierung gehören Ämter wie: Sozialamt, Jugendamt, Schulamt. Kommunalpolitische Hebel der Macht liegen eher bei den Ämtern der marktgetriebenen Sozialraumorientierung und weniger bei den Ämtern der hilfreichen Sozialraumorientierung.

Wir stehen im Feld der hilfreichen Sozialraumorientierung und sollten uns nicht blauäugig verhalten. Es ist deshalb nötig aus unserer Sicht Folgendes zu leisten:

- Wir brauchen eine klare Vision. »Warum ist es nützlich für die Stadt, die Erfahrungen aus den Feldern der Sozial- und Jugendhilfe zu nutzen für die marktorientierte Sozialraumorientierung?«

- Entsprechende Aktivitäten des Überzeugens und des Einmischens müssen zunächst von unserem Bereich ausgehen.

- Die Trennung von kommunaler Lebenswelt und örtlicher Zuständigkeit muss praktisch überwunden werden. Was das bedeuten kann, sehen wir später.

\section{Zweitens: Entschlossenheit auf kommunaler Ebene}

Wenn eine kommunale soziale Stadtentwicklung politisch gewollt ist, dann kann eine marktorientierte Sozialraumorientierung nicht auf das Wissen des Allgemeinen Sozialen Dienstes verzichten. Entsprechende Wissenstatbestände müssen dann integriert werden. In diesem $\mathrm{Zu}$ sammenhang muss man zwei Aufgaben lösen:

- die »Eingemeindung « des örtlichen Trägers der Sozialund Jugendhilfe (Landkreis) und

- die »Interpenetration « zwischen der marktgetriebenen Sozialraumorientierung und der hilfreichen Sozialraumorientierung in der jeweiligen Kommunalverwaltung.

Zur »Eingemeindung « gibt es unterschiedliche rechtliche und organisatorische Möglichkeiten:

- Die kreisangehörige Stadt unterhält einen eigenen Allgemeinen Sozialen Dienst und nimmt die Aufgaben des örtlichen Trägers der Jugend- und Sozialhilfe in Delegation wahr.

- Beide betreiben einen gemeinsamen Sozialdienst (Hilfe aus einer Hand vor Ort).

- Der Landkreis schafft in der entsprechenden Kommune eine Außenstelle.

In allen Fällen - auch in der vierten Konstellation: Hilfesuchende müssen entweder ins Landratsamt oder zu einer Außenstelle, die nicht in ihrem Wohnort ist - bedarf es eines lebensraumorientierten Datenaustausches und eines entsprechenden Netzwerkmanagements. In jedem Fall muss die sozialraumbewusste Kommune entschlossen handeln und es nicht zulassen, dass ihre eigenen Handlungsmöglichkeiten (im Sinne des Verständnisses der Gemeindeordnung) geschwächt oder entpolitisiert werden.
Bezogen auf das Thema »Interpenetration « geht es vor allem um die Gestaltung einer »inneren Angelegenheit« der Kommune. Gemeint ist eine gelingende Kooperation zwischen den Ämtern, die in den unterschiedlichen »Wahrheitsbereichen « arbeiten: marktgetriebene Sozialraumorientierung und hilfeorientierter Sozialraumorientierung.

\section{Drittens: Landkreisplanung als Dienstleistung für kreisangehörige Kommunen}

Es geht hier zunächst um die inhaltliche Orientierung der Jugendhilfe und der Sozialplanung des örtlichen Trägers - also des Landkreises. Erfahrungsgemäß kümmert sie sich vor allem um jene Felder, die mit der eigenen Kostenträgerschaft verbunden sind.

Natürlich gibt es auch Beispiele für Sozialraumorientierung. Aber: Die Welt, die dort durch das Spiel von Nachfrage, Angebot, Kosten und Träger modelliert wird, hat nur teilweise etwas zu tun mit der integrierten Lebenswelt der Hilfesuchenden. Settings der Hilfe sind in der Regel Settings der Aussonderung. Beispiele: Heimversorgung substituiert oft Angebote im Normalmarkt des Wohnens. Das lässt sich in den Bereichen Jugendhilfe, Altenhilfe, Behindertenhilfe und Wohnungslosenhilfe nachweisen.

Jugendhilfe und bzw. Sozialplanung, die auf solche Effekte aufmerksam macht, kann Impulse setzen für die Entwicklung kommunaler Wohnungspolitik. Das würde allerdings für die Landkreisplanung bedeuten, dass sie - Effekte der von ihr finanzierten Hilfe systematisch auswertet und

- entsprechende Erkenntnisse im Rahmen von Dienstleistungen an die Kommunalplanung weitergibt.

Viele Landkreise haben ihre Planungsstäbe weder qualitativ noch quantitativ entsprechend ausgestattet. Hier gibt es aus meiner Sicht einen erheblichen Qualifizierungsbedarf.

\section{Viertens: Wissensbilanzierung und Netzwerkmanagement}

In der Wirtschaftsförderung gibt es eine Praxis, die als »lernende Region « beschrieben wird. Hier kann eine »Wissensbilanzierung« eine Rolle spielen. Was ist damit gemeint? - Unternehmen gründen ein Netzwerk, in dem die Kooperation zwischen den Unternehmen verbessert werden soll.

- Jedes Unternehmen bilanziert sein Wissen in unterschiedlichen Wissensarten, beispielsweise: marktfähiges Wissen, Mitarbeiterkompetenzen, Netzwerkverbindungen.

- Basierend auf diesem Unternehmensspezifischen Wissensbilanzen werden im Sinne der Optimierung von Angebot und Nachfrage im Netzwerk Verträge geschlossen, wenn eine Win-win-Konstellation zu erwarten ist.

- Der eingetretene wechselseitige Ertrag wird bewertet und gegebenenfalls zur Verbesserung bestehender Verträge oder zum Abschluss neuer Verträge genutzt.

- Die gesamte Netzwerkpraxis ist im Netzwerk öffentlich und definiert die Netzwerkgrenzen. Nach Außen werden spezifische Informationen nicht weitergegeben. 
Es scheint mir sinnvoll, solche Strategien zu überprüfen im Hinblick auf ihre Übertragbarkeit für unseren Bereich. Im Landkreis gibt es

- viele Angebote, die sich gegenseitig behindern

- viele Parallelstrukturen

- viel Unwissen voneinander

- zu wenig Erfahrungstransfer zwischen den Kommunen.

Effekte, die oft verstärkt werden durch mangelhafte Abstimmung zwischen kommunaler Zuständigkeit nach der Gemeindeordnung und örtlicher Zuständigkeit nach den entsprechenden Sozialgesetzbüchern.

\section{Fünftens: Arbeit mit Projektstrukturen}

Wenn es einer Kommune im Landkreis darum geht, das "gemeinsame Wohl ihrer Einwohner zu erhalten bzw. zu verbessern « durch eine nachhaltige Politik der Sozialraumentwicklung, dann kann sie sich nicht auf die Erfahrung des Landkreises als örtlichem Träger der Jugend- und Sozialhilfe und seinem Verständnis und seiner Erfahrung mir Sozialraumorientierung verlassen. Die Kommune muss einen eigenen Rahmen schaffen, der garantiert, dass das beachtet und genutzt wird, was der Landkreis weiß und der dort in der Regel ressortierende Allgemeine Soziale Dienst. Oft arbeitet eine solche Kommune mit einer Projektstruktur. Mit Projekt ist hier ein Vorhaben kommunaler Planung einer großen Kreisstadt in einem Landkreis gemeint. Unser Beispiel stammt aus Schwäbisch Gmünd. Hier ging es um die Fortentwicklung der »offenen Kinder- und Jugendarbeit«. Wie eine solche Struktur visualisiert werden kann, wird aus der Abbildung »Projektstruktur« deutlich (vgl. Seite 19). Anschlüsse für das Wissen des Allgemeinen Sozialen Dienstes werden über die Arbeitsgruppen und den Planungsbeirat organisiert.

Im Mittelpunkt der Arbeit mit solchen Projektstrukturen steht eine Steuerungsgruppe. In diesem Falle ressortierte die Steuerungsgruppe beim Sozialamt. Die Steuerungsgruppe organisiert ihre Arbeit in vier Handlungskreisen. Diese sind:

- sozialräumliche Berichterstattung

- Evaluation der Angebote unter einer dreifachen Perspektive (sozialraumspezifisch, angebotsspezifisch und gesamtstädtisch)

- Umsetzung der Evaluationsergebnisse für die kommunale Planung

- Entscheidungsvorbereitung für beschließende Gremien

In der amtsinternen Steuerungsgruppe müssen Qualifikationen vorhanden sein zu den Bereichen: qualitative Berichterstattung, Evaluation, Planung, Gremienarbeit und Politikberatung.

\section{Sechstens: integrierte Datensätze}

Ein großes Problem der kommunalen Planung besteht oft im Mangel relevanter Daten, die die Lebenslage der Einwohnerinnen und Einwohner abbilden können. Das hängt u. a. mit den unterschiedlichen gesetzlichen $\mathrm{Zu}$ ständigkeiten zusammen. Ich will das beispielhaft an der Zielgruppe der unter 25-jährigen Menschen ( $U$ U 25«) deutlich machen.
Wenn eine Kommune im Landkreis etwas verbessern wollte im Bereich der Hilfen für junge Menschen in Berufsnot, dann befindet sie sich - wie die jungen Menschen selbst - in einem Dschungel. Relevant sind hier mindestens

- für Ausbildung: SGB III

- für Grundsicherung: SGB II

- für Jugendhilfe: SGB VIII (z. B. § 13)

- für Sozialhilfe: SGB XII (z. B. § 67)

- Berufsschule: Berufsschulgesetz (Land fürs Personal und Kommune als Träger).

Hier überlagern sich mindestens fünf Rechtskreise. In München hat man für die betroffenen Jugendlichen einen »Reiseführer durch den Dschungel« erstellt und verteilt. Da zwischen den Rechtssystemen aufgrund der Datenschutzbestimmungen Grenzen bestehen, ist es für die kommunale Planung extrem schwer, an die Daten zu kommen, die sie für eine angemessene Entwicklung der Jugendberufshilfe braucht. Notwendig wären integrierte Daten als Grundlage für eine Gesamtplanung. Hier gibt es einen erheblichen Entwicklungsbedarf auf inhaltlicher Ebene (»Was wird für eine angemessene Planung vor Ort wirklich gebraucht?«) und auf rechtlicher Ebene (»Welche rechtlichen Grundlagen müssen entwickelt werden, die es ermöglichen, planungsrelevante Datensätze quer zu den Rechtskreisen zu generieren?«).

Aus dem derzeitigen Dilemma kommen die Kommunen nur heraus, wenn sie Zusatzerhebungen durchführen; das wird wenig gemacht, weil es zum Teil sehr kostenintensiv ist.

\section{Resümee}

Ich komme zum Schluss und will eine kurze Zusammenfassung anbieten:

- Der Allgemeine Soziale Dienst hat viele Erfahrungen, was die Sozialraumorientierung vor Ort angeht. Dieses Wissen sollte genutzt werden.

- Probleme gibt es dort, wo die kommunale Verantwortung nach der Gemeindeordnung getrennt wird von örtlicher Zuständigkeit für Sozial- und Jugendhilfe entsprechend den jeweiligen Sozialgesetzbüchern.

- Aktivitäten zur Überwindung dieser Trennung müssen von den Kommunen ausgehen. Dies kann in vielfältiger Weise geschehen: durch Übertragung, Bürogemeinschaften, integrierte Dienste. In jedem Fall braucht die Kommune eine Projektstruktur für die eigene kommunale Planung, die alle relevanten Wissenstatbestände (auch die des Allgemeinen Sozialen Dienstes) zusammenfasst und ein Netzwerkmanagement organisiert.

- Bei der Sozialraumentwicklung sind die zwei Logiken der Planung und der Steuerung zu beachten; einerseits die marktgetriebene Sozialraumorientierung und andererseits die hilfeorientierte Sozialraumorientierung. Hier treten regelmäßig Konflikte auf. Insofern muss die Kommune interne Planung als die Organisation von »Wertkonflikten« verstehen.

- Vor Ort fehlen integrierte Datensätze. Hier entsteht ein erheblicher Entwicklungsbedarf in methodischer und rechtlicher Hinsicht.

- Die Planungsabteilungen auf Landkreisebene - sofern es diese überhaupt gibt - müssen sich vor allem in folgende Richtung entwickeln: Auswertung ihrer eigenen Daten auf kommunale Planungsrelevanz und Unterstützung der kommunalen Planung durch entsprechende Dienstleistung. 